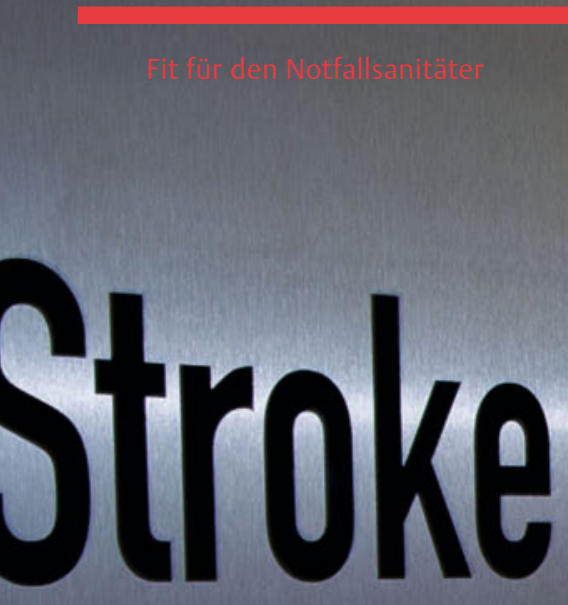

\title{
Schlaganfall
}

\section{Ergänzungsprüfung - Das sollten Sie wissen}

retten! macht Sie fit für den Notfallsanitäter: In jeder Ausgabe arbeiten wir anhand eines Fallbeispiels einen interessanten Einsatz algorithmenkonform auf. Anhand von exemplarischen Fragen zu erweiterten Notfallmaßnahmen, Kommunikation und Rahmenbedingungen können Sie sich auf die Ergänzungsprüfung vorbereiten - egal, in welchem Bundesland Sie arbeiten.

Rico Kuhnke - Wolfgang von Meißner

Fallbeispiel Einsatzmeldung: „Hilfloser männlicher Patient in einer Wohnung“. Am Eingang wartet schon eine rüstige ältere Dame auf das Rettungsteam. Die Frau wirkt aufgelöst und führt die Helfer ins Schlafzimmer. Dabei schildert sie, dass ihrem Mann bereits beim Frühstück unwohl und schwindelig gewesen sei. Er habe sich deshalb noch ein wenig ausgeruht. Nach einigen Stunden habe sie sich Sorgen gemacht und nach ihrem Mann geschaut. Da habe er bereits nicht mehr richtig mit ihr gesprochen.
Situation vor Ort Der Patient liegt im Bett und nimmt bei Eintreten des Teams mit den Augen Kontakt auf. Sofort fallen der hängende Mundwinkel und ein leichter Speichelfluss auf. Der Patient bemüht sich zu sprechen, stammelt aber nur unzusammenhängende Sätze. Während einer der Kollegen das Material vorbereitet, beginnt der andere mit der gezielten Untersuchung nach dem ABCDE-Schema.

Beruhigendes Zureden Immer wieder versucht der Patient zu sprechen und wirkt zunehmend verzweifelter. Es gelingt ihm nicht, zusammenhängende Sätze zu bilden. Die Sprache ist verwaschen, und er leidet unter nachhaltigen Wortfindungsstörungen. Das Sprachverständnis scheint nicht 
gestört zu sein. Der Untersucher nimmt sich Zeit, den Patienten zu beruhigen, und formuliert geschlossene Fragen, auf die der Patient nur mit ja und nein antworten muss.

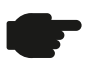

Eine Übersicht von rettungsdienstlichen Merkhilfen haben wir in dem Plakat „Fit für den Einsatz? Das sollten Sie wissen!“ zusammengefasst. Von ABCDEüberSAMPLER+S bis zum FAST-Schema bietet es eine gute Übersicht zur Vorbereitung auf Ihre Ergänzungsprüfung. Das Plakat können Sie kostenfrei unter o www.thieme.de/retten-poster bestellen.

A-irway Der Patient reagiert prompt, und die Atemwege sind frei.

B-reathing Die Atmung ist leicht beschleunigt, aber unauffällig. Die Pulsoxymetrie zeigt einen $\mathrm{SpO}_{2}$ von $96 \%$. Da sich bereits jetzt die Verdachtsdiagnose Apoplex aufdrängt, erhält der Patient $51 \mathrm{O}_{2} / \mathrm{min}$ über eine Nasensonde. Eine kurze Auskultation ergibt keinen pathologischen Befund.

C-Circulation Der Puls lässt sich peripher sehr gut tasten, er liegt bei ca. 120/min und ist regelmäßig. Der Blutdruck ist mit $150 / 90 \mathrm{mmHg} z$ war etwas hyperton, erfordert aber präklinisch keine Therapie. Das EKG ergibt außer einer Sinustachykardie keinen pathologischen Befund.

D-isability Der Patient ist wach und öffnet spontan die Augen. Er kann nur sehr eingeschränkt reden - die Kommunikation beschränkt sich weitestgehend auf Nicken und Kopfschütteln. So antwortet er auf die Frage „Wie fühlen Sie sich jetzt?“ sehr stockend und angestrengt: „Äh, es geht kein und, äh, nicht aufstand, äh, Sprech schwer, äh.“ Als einer der Helfer ihm einen Kugelschreiber zeigt, kann der Patient diesen nicht richtig benennen. Auch das Wiederholen von Sätzen ist nur sehr eingeschränkt möglich. Insgesamt wird der neurologische

Tab. 1 Glasgow Coma Scale (GCS) für Erwachsene

\begin{tabular}{llll} 
Punkte & Augen öffnen & verbale Kommunikation & motorische Reaktion \\
\hline 6 & - & - & befolgt Aufforderungen \\
\hline 5 & - & konversationsfähig, orientiert & gezielte Schmerzabwehr \\
\hline 4 & spontan & konversationsfähig, desorientiert & ungezielte Schmerzabwehr \\
\hline 3 & auf Aufforderung & $\begin{array}{l}\text { unzusammenhängende Wörter } \\
\text { (Wortsalat) }\end{array}$ & auf Schmerzreiz Beugesynergismen \\
\hline 2 & aufSchmerzreiz & unverständliche Laute & auf Schmerzreiz Strecksynergismen \\
\hline 1 & keine Reaktion & keine verbale Reaktion & keine Reaktion auf Schmerzreiz
\end{tabular}

Status mit einem GCS-Wert ( $\bullet$ Tab. 1) von 13 bestimmt. Die Pupillen sind isokor und reagieren prompt auf Licht. Der Blutzucker ist mit $140 \mathrm{mg} / \mathrm{dl}$ erhöht, die Temperatur mit $36,8^{\circ} \mathrm{C}$ unauffällig.

E-Exposure Die körperliche Untersuchung ergibt eine deutliche Hemiparese rechts.

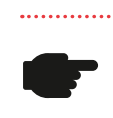

Mit dem FAST-Schema lässt sich rasch ein Verdacht auf einen apoplektischen Insult bestätigen bzw. ausschließen.

$\triangleright$ F-ace Bitten Sie den Patienten, zu grimassieren. Lassen Sie ihn die Lippen spitzen oder die Backen blähen, achten Sie auf hängende Mundwinkel und Halbseitenlähmungen.

$\triangleright$ A-rms Nehmen Sie die gestreckten Arme Ihres Patienten und bitten Sie ihn, diese ausgestreckt zu halten (Armhalteversuch). Geben Sie ihm dann beide Hände (Kreuzgriff) und lassen Sie ihn diese fest drücken. Achten Sie auf Halbseitenschwächen.

$\triangleright$ S-peech Bitten Sie den Patienten, einen Gegenstand zu benennen, Sätze nachzusprechen oder auf eine Frage zu antworten. Achten Sie auf Sprachstörungen (Aphasien).

$\triangleright$ T-ime Bei positivem Befund ist ein rascher Transport in eine Stroke Unit anzustreben („time is brain“). Je nach Klinik beginnt man eine Thrombolyse noch max. 4,5-6h nach den ersten Symptomen.
SAMPLER-Schema Während sich ein Kollege um den Patienten kümmert, bereitet der andere den venösen Zugang vor. Währenddessen befragt er die Ehefrau des Patienten und vervollständigt die Anamnese mithilfe des SAMPLER-Schemas:

- S-ymptome Hängender Mundwinkel und Speichelfluss, Hemiparese rechts, Wortfindungsstörungen.

- A-llergien Allergien sind nicht bekannt.

- M-edikamente Die Ehefrau gibt an, dass ihr Mann unter Altersdiabetes leidet und orale Antidiabetika einnimmt.

- P-atientengeschichte Der Patient hat in seiner Vorgeschichte keine relevanten Erkrankungen. Die Ehefrau beschreibt, dass er seit Jahren über zunehmende Kurzatmigkeit und hohen Blutdruck klage, sich aber weigere, zum Arzt zu gehen.

- L-etzte Mahlzeit Zum Frühstück habe er ein gekochtes Ei und ein Marmeladenbrot gegessen.

- E-reignis Die ersten Symptome sind vor ca. $3 \mathrm{~h}$ aufgetreten und haben sich seitdem zunehmend verschlechtert.

- R-isikofaktoren Der Patient ist 63 Jahre alt und leidet unter einem Altersdiabetes sowie einer deutlichen Adipositas mit chronischer Hypertonie.

Reevaluation Das Team wiederholt nun noch einmal die Punkte A, B und C. Die Vitalfunktionen sind unverändert. Aufgrund der Anamnese entscheidet sich das Team für einen raschen Transport in die Klinik und meldet den Patienten direkt auf der nächstgelegenen Stroke-Unit mit der Ver- 
dachtsdiagnose „ischämischer Insult“ an. Um eine schnellstmögliche weiterführende Therapie in der Klinik zu ermöglichen, erfolgt der Transport mit Sonderrechten. Dabei wird der Patient in leichter $\left(30^{\circ}\right)$ Oberkörperhochlage gelagert, wobei das Team darauf achtet, dass der Kopf nicht angewinkelt ist.

\section{Der Algorithmus}

Vorgehen bei Schlaganfall Das Team im beschriebenen Fall nutzt den „Algorithmus Schlaganfall“ aus Ostwestfalen-Lippe ( Abb. 1). Wie die meisten Algorithmen beginnt auch er erst mit der Verdachtsdiagnose. Bis dahin arbeitet das Team konsequent nach $A B C D E-S c h e m a$. Infoboxen zu den typischen Symptomen für einen Schlaganfall oder mit Hinweisen auf die notwendigen Basismaßnahmen ergänzen den Algorithmus.

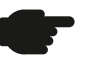

Sollten Sie in Ihrem Rettungsdienstbereich ein abweichendes Konzept nutzen, arbeiten Sie die Unterschiede heraus. Überlegen Sie sich, wie der Einsatz nach Ihrem Algorithmus ablaufen müsste.

Sensibler Umgang mit dem Patienten Gleich zu Beginn stellt das Team eine schwere Sprachstörung fest: Der Patient kann sich nicht mitteilen und hat massive Angst. Dies erklärt auch den hohen Puls von 120/min. Ein ruhiges Auftreten der Helfer wirkt nicht nur beruhigend auf das Empfinden des Patienten, sondern kann auch die Kreislaufwerte normalisieren. Durch die geschlossenen Fragen ist der Patient in der Lage, Auskunft zu geben - die sprachliche Unfähigkeit fällt nicht weiter ins Gewicht. Während man sonst offene Fragen beim Erheben der Anamnese favorisiert, sind die geschlossenen in diesem Fall sinnvoll.

\section{Präklinischer Algorithmus - Schlaganfall}
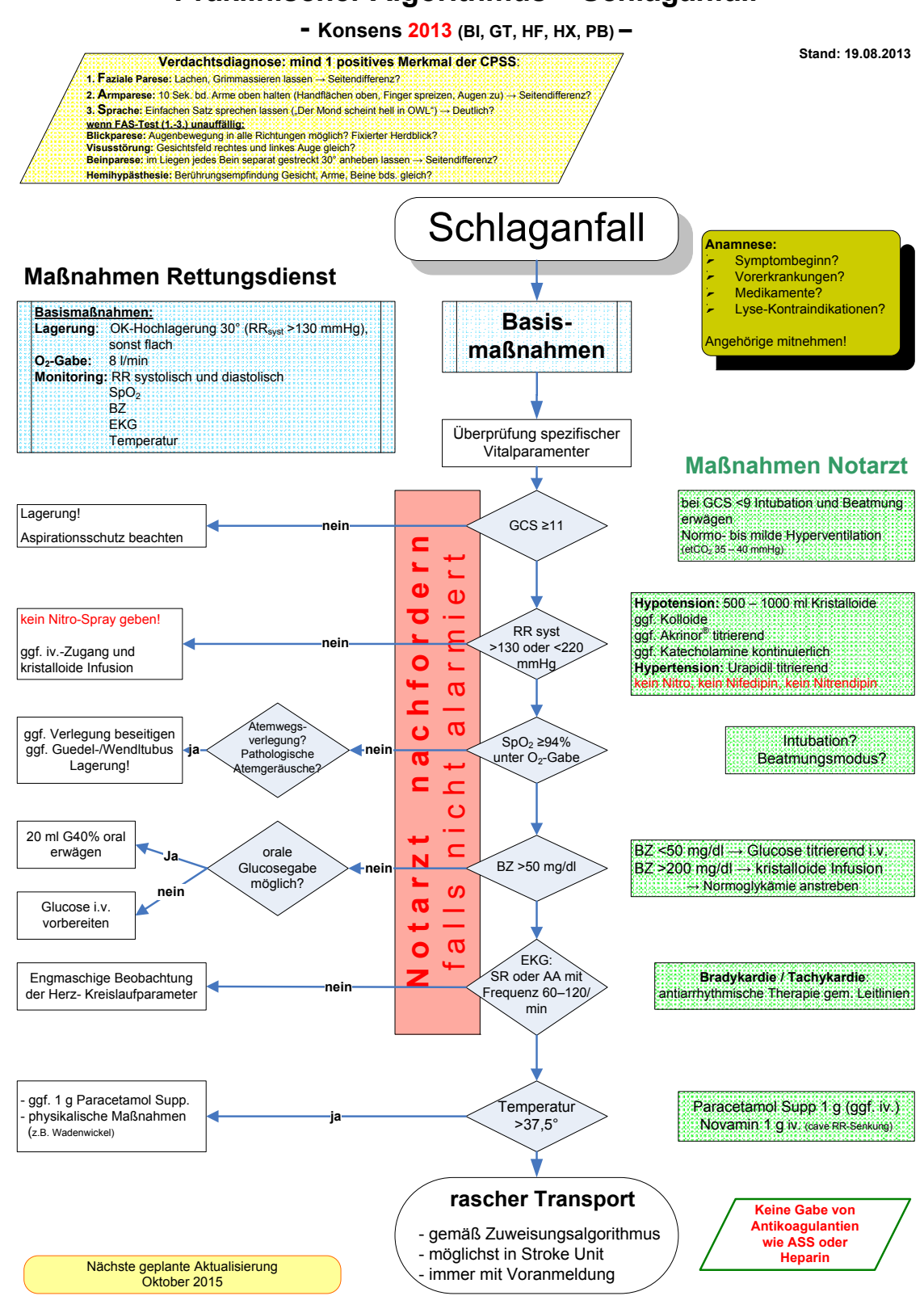

Abb. 1 Gemeinsamer präklinischer Algorithmus Schlaganfall in Ostwestfalen-Lippe (OWL), Stand 19.8.2013. Die geplante Aktualisierung verschiebt sich auf Ende 2016 (Details dazu im Kommentar auf S. 263). Ziel ist eine Versorgungszeit < 20 min und insg. eine Prähospitalzeit < 60 min. AA: absolute Arrhythmie, BZ: Blutzucker, CPSS: Cincinnati Prehospital Stroke Scale, etCO $\mathrm{C}_{2}$ : endtidaler $\mathrm{CO}_{2}$-Druck, GCS: Glasgow Coma Scale, OK: Oberkörper, RR: Blutdruck, $R_{\text {syst }}$ : Blutdruck systolisch, $\mathrm{SpO}_{2}$ : Sauerstoffsättigung, SR: Sinusrhythmus.

Aphasie Bei der hier festgestellten Sprachstörung handelt es sich vermutlich um eine sog. Broca-Aphasie. Sie ist durch angestrengte, stockende Sprache gekennzeichnet - Kommunikation ist schwer oder nur sehr eingeschränkt möglich. Während das
Nachsprechen von Sätzen und das Benennen von Gegenständen gestört sind, bleibt das Sprachverständnis weitgehend normal. Dies erlaubt dem Rettungsdienstteam, Antworten auf gezielte Fragen zu erhalten. 
Denken Sie bei Sprachstörungen daran, dass das Sprachverständnis gestört sein kann. Verschaffen Sie sich daher zu Beginn der Untersuchung mit einer Kontrollfrage Sicherheit.

Überprüfung und Sicherung der Vitalfunktionen Sehr schnell wird dem Team klar, dass die Vitalfunktionen des Patienten im Normbereich liegen und keine erweiterten Maßnahmen notwendig machen. Der Schwerpunkt der Anamnese liegt auf dem neurologischen Status (Disability) und der Klassifizierung mithilfe der Glasgow Coma Scale (GCS). Alternativ kann man das vereinfachte WASB-Schema (Wach, Ansprache, Schmerz, Bewusstlos) nutzen. Für die Akutversorgung reicht diese Klassifizierung aus. Um allerdings minimale Veränderungen des neurologischen Status erfassen zu können, ist die GCS deutlich besser geeignet. Im beschriebenen Fall ist es in erster Linie die eingeschränkte verbale Reaktion, die sich in dem verminderten GCS-Wert widerspiegelt. Ob man diese mit 3 oder 4 Punkten beurteilt, kann man diskutieren - macht aber für die weitere Behandlung keinen Unterschied.

Verdachtsdiagnose Schlaganfall Die Verdachtsdiagnose Schlaganfall drängt sich bereits früh auf. Der hängende Mundwinkel, Speichelfluss, nachhaltige Sprachstörungen (Aphasie) sowie die ausgeprägte rechtsseitige Muskelschwäche (Hemiparese) sind deutliche, aber noch nicht eindeutige Symptome für einen Schlaganfall (Apoplex). Zum Ausschluss einer Hypoglykämie als Differenzialdiagnose bestimmt man daher den Blutzuckerspiegel (BZ).

Blutzucker Die frühe BZ-Messung ( $\bullet$ Abb. 2) bei Bewusstseinsstörungen kann nicht genug betont werden. Immer noch kommt es vor, dass sich ein „Schlaganfall“ in der Notaufnahme als Hypoglykämie herausstellt. Aus diesem Grund muss man vor einer Diagnosestellung immer den Blutzuckerspiegel messen. Im beschriebenen Fall ist der BZ nicht akut behandlungsbedürftig, aber mit $140 \mathrm{mg} / \mathrm{dl}$ langfristig zu hoch. Im Verlauf sollte hier z. B. der Hausarzt den BZ überprüfen - etwa durch Bestimmung des Glykohämoglobins $\mathrm{HbA}_{1 \mathrm{c}}$ („Langzeit-Blutzucker“) - und ggf. die Medikamentendosis anpassen.

Behandlung des Blutdrucks Der Blutdruck liegt im Normbereich, und der Algorithmus sieht keine weiterführende medikamentöse Therapie vor. Die in den farbig hinterlegten Feldern enthaltenen Handlungshinweise geben nur eine grobe Hilfestellung zur notwendigen Medikation bei abweichenden Werten. Dies macht ein hochqualifiziertes Rettungsdienstpersonal notwendig, das in der Medikamentenapplikation geschult und geprüft ist.

Anmeldung Stroke Unit Entsprechend den Leitlinien der Deutschen Gesellschaft für Neurologie (DGN) zur Akuttherapie des ischämischen Schlaganfalls soll eine i.v. Thrombolyse spätestens 4,5 h nach Symptombeginn starten [1]. Da die ersten Symptome beim Patienten vor ca. $3 \mathrm{~h}$ aufgetreten sind, darf das Team hier keine Zeit verlieren. Die Anmeldung in einer Stroke Unit verkürzt die Aufnahmezeit, und es kann sofort eine kranielle Computertomographie (CCT) zum Ausschluss einer zerebralen Blutung vorgenommen werden. Laut DGN sind $80 \%$ der jährlich 250000 Schlaganfälle ischämischer Ursache. Nur bei $20 \%$ der Fälle ist eine

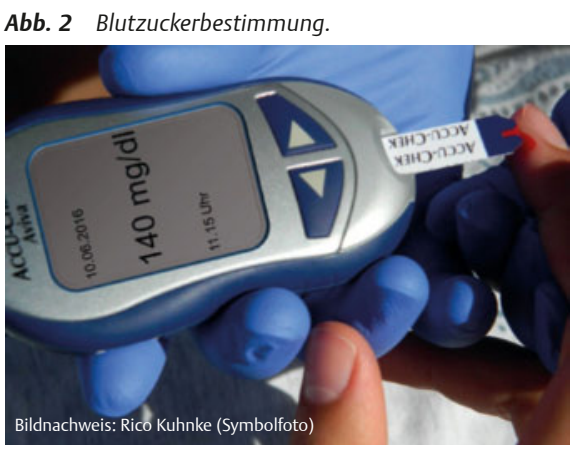

\section{Unterstützen Sie uns!}

Für die Rubrik „Fit für den Notfallsanitäter“ verwenden wir die unterschiedlichsten Algorithmen und SOPs aus dem gesamten Bundesgebiet. Deshalb würden wir an dieser Stelle gern auch einen Ihrer Algorithmen vorstellen. Unterstützen Sie uns! Senden Sie uns Ihre regionalen Algorithmen und SOPs an: retten@thieme.de.

Blutung die Ursache. Die Entscheidung für einen raschen Transport und das Fahren mit Sonderrechten ist vor dem Hintergrund „time is brain“ absolut gerechtfertigt.

\section{NOTFALLMEDIZIN}

\section{(2) Beschreiben Sie die Ursachen für ei- nen apoplektischen Insult und die Möglichkeiten zu deren Einteilung. Begründen Sie, warum man bei der präklinischen Behandlung einer hy- pertensiven Krise die systolischen Werte nicht $<180 \mathrm{mmHg}$ senken soll.}

$\int$ Ursachen des Schlaganfalls Bei einem apoplektischen Insult kommt es zu einer Unterversorgung von Hirngewebe mit nachfolgendem Funktionsausfall. Der Funktionsausfall äußert sich typischerweise in einem neurologischen Defizit, das die Funktionen betrifft, die in der betroffenen Region gesteuert werden.

- In $>80 \%$ ist die Ursache eine Minderdurchblutung infolge einer akuten Embolie oder gefäßverengender Prozesse.

- Deutlich seltener ist eine zerebrale Blutung Ursache für einen Schlaganfall.

Klassifizierung Während man zerebrale Blutungen i.d.R. nach der Blutungslokalisation klassifiziert, erfolgt die Einteilung einer zerebralen Ischämie nach deren Verlauf (TIA: transitorische ischämische Attacke, PRIND: prolongiertes reversibles ischämisches neurologisches Defizit, Progressive Stroke, Complete Stroke). Für den Rettungs- 
dienst ist dies aber nicht weiter relevant, da er meist wenige Stunden nach den ersten Symptomen beim Patienten eintrifft.

Blutdrucksenkung bei hypertensiver Krise Grund für eine hypertensive Krise ist i.d.R. eine Ischämie. Der Körper reagiert auf die Sauerstoffunterversorgung mit einer reflektorischen Erhöhung des Blutdrucks, um eine Mindestperfusion der betroffenen Regionen zu erreichen - man spricht von einer „Bedarfshypertonie“. Ziel der präklinischen Therapie ist es, den Blutdruck so weit zu senken, dass

- eine Gefäßschädigung mit nachfolgender Blutung vermieden wird und

- gleichzeitig der Blutdruck hoch genug bleibt, um die ischämischen Bereiche weiter mit Blut zu versorgen.

Die Autoregulation des Gehirns ermöglicht zwar einen weitgehend konstanten Blutfluss - jedoch nur bei systolischen Drücken von ca. 100-200 mmHg ( Infobox online).

Mögliche weitere Fragen:

Erläutern Sie die Möglichkeiten einer medikamentösen Blutdrucksenkung.

Welche Maßnahmen ergreifen Sie laut Algorithmus zur Stabilisierung des Blutdrucks bei systolischen Werten $<130 \mathrm{mmHg}$ ?

Beschreiben Sie grob die diagnostischen und therapeutischen Schritte nach Aufnahme in der Klink.

\section{KOMMUNIKATION}

Erläutern Sie den Unterschied von Sprech- und Sprachstörungen. Beschreiben Sie die Besonderheiten der verschieden Aphasieformen.

! Definitionen Unter einer Aphasie versteht man eine Sprachstörung. Dabei ist die Wortbildung im zentralen Nervensystem (ZNS) gestört, während bei einer
Sprechstörung der motorische Ablauf der Lautbildung beeinträchtigt ist.

Charakteristika der Broca-Aphasie Bei einer Broca-Aphasie (motorischen Aphasie) handelt es sich um eine Einschränkung bzw. den vollständigen Verlust der Ausdrucksfähigkeit in Sprache und Schrift.

- Das Sprechen wirkt sehr angestrengt und ist meist verlangsamt.

- Die Patienten machen immer wieder Pausen und haben große Mühe, die richtigen Worte zu finden.

- Häufig verwechseln sie beim Sprechen Silben und Buchstaben.

Das Sprachverständnis ist i.d.R. nicht betroffen, und die Patienten sind in der Lage, auf Fragen zu antworten. Die Patienten erleben die Situation als äußerst bedrohlich, da sie zwar wissen, was sie gerne sagen möchten, dies aber nur schwer oder gar nicht können.

Wernicke-Aphasie Dahingegen wirkt die Sprache bei einem Patienten mit einer

Wernicke-Aphasie (sensorische Aphasie) sehr flüssig.

- Die Sprachmelodie ist unauffällig, allerdings ist die Sprache inhaltsarm.

- Die Eigenwahrnehmung bei den Patienten für das eigene Sprachdefizit ist nachhaltig eingeschränkt.

- Ebenso ist das Sprachverständnis gestört, und die Betroffenen verstehen Fragen nur eingeschränkt oder gar nicht.

Weitere Aphasien Darüber hinaus werden in der Literatur noch globale Aphasien und amnestische Aphasien beschrieben.

- Bei einer globalen Aphasie ist die Sprachbildung auf Automatismen und Floskeln beschränkt,

- die amnestische Aphasie äußert sich in reinen Wortfindungsstörungen.

In der aktuellen Diskussion geht man mehr und mehr weg von den klassischen Einteilungen hin zu detaillierten Einzelfallbeschreibungen.
Mögliche weitere Fragen:

$?$ Welche Ängste und Sorgen belasten einen Patienten mit akuter Sprachstörung? Reflektieren Sie die Bedürfnisse von Patienten und Angehörigen in der beschriebenen Situation.

\section{RAHIMENBEDINGUNGEN}

2 Immer wieder kommt es im Rettungsdienst zu eigentlich strafbaren Handlungen - im beschriebenen Fall z. B. zum Tatbestand der Körperverletzung beim Legen eines i.v. Zugangs. Was wird durch das Strafrecht geregelt und welcher Systematik folgt die Feststellung einer strafbaren Handlung?

! Aufgabe des Strafrechts Das Strafrecht regelt mithilfe von Verboten das Verhalten der Bürger untereinander.

- Ziel ist es, den gewünschten Umgang der Menschen in der Gesellschaft zu regeln. Dabei unterscheidet der Gesetzgeber zwischen Tatbeständen durch aktives Tun und solchen durch Unterlassen.

- Verstöße gegen die aufgestellten Normen werden mit Geld- oder Freiheitsstrafen belegt.

- Zudem gibt das Strafrecht die Möglichkeit von sog. Nebenstrafen. Dabei handelt es sich um einschränkende Maßnahmen (Berufsverbot, Fahrverbot) für den Täter

Erhält die Exekutive (Polizei und Staatsanwaltschaft) Kenntnis von einer Straftat, ist sie zur Ermittlung verpflichtet.

Strafbar gehandelt oder nicht? Das Feststellen einer strafbaren Handlung folgt immer der gleichen 3-stufigen Systematik: Tatbestand, Rechtswidrigkeit, Schuld.

1. Tatbestand $\mathrm{Zu}$ Beginn ist zu prüfen, ob es sich um einen Tatbestand im Sinne des Strafgesetzbuchs handelt. Strafbar ist dabei nicht nur die Erfüllung des objekti- 
ven Tatbestands, wie z.B. Körperverletzung oder Sachbeschädigung, sondern auch ein subjektiver Tatbestand, wie z. B. Motive, Absichten und Vorsätze.

2. Rechtswidrigkeit Ist der Tatbestand erfüllt, ist zu prüfen, ob dieser auch wirklich rechtwidrig ist. Grundsätzlich ist natürlich davon auszugehen, dass jeder Tatbestand dieses Kriterium (objektive Vorwerfbarkeit der Tatbestandsverwirklichung) erfüllt. Es gibt allerdings Rechtfertigungsgründe, die dazu führen, dass ein Tatbestand nicht rechtswidrig ist. Dies sind bspw. neben der Einwilligung bzw. mutmaßlichen Einwilligung des Patienten die Notwehr und der rechtfertigende Notstand. Bei der Einwilligung gilt aber einschränkend: Der Tatbestand darf nicht gegen die guten Sitten verstoßen.

3. Schuld Im letzten Schritt prüft man, ob für den Tatbestand auch die Schuld (subjektive, persönliche Vorwerfbarkeit der Tatbestandsverwirklichung) erfüllt ist. In diesem Zusammenhang relevant sind entschuldigende Faktoren, wie die
Schuldunfähigkeit oder evtl. eine verminderte Schuldfähigkeit.

Sind alle 3 Stufen durchlaufen, ordnet der Gesetzgeber eine bestimmte Rechtsfolge an (Strafe oder Freispruch / Einstellung des Verfahrens).

\section{Mögliche weitere Fragen:}

\section{Nennen Sie Beispiele aus der Praxis zur Untermauerung Ihrer Argumen- tation. \\ 2 Welche Rechtfertigungsgründe ken- nen Sie? Nennen Sie Beispiele aus der Praxis.

Was sind Gründe für eine Schuldun-
fähigkeit bzw. eine verminderte
Schuldunfähigkeit des Täters?

\section{Infos im Internet}

Das Literaturverzeichnis und die Infobox zu diesem Beitrag finden Sie online.

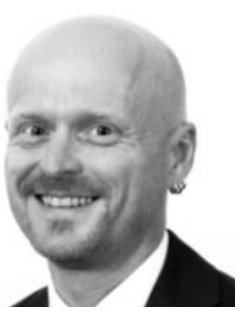

Rico Kuhnke ist Schulleiter der DRK-Landesschule Baden-Württemberg. Er war viele Jahre als Lehrrettungsassistent tätig und ist Notfallsanitäter. Er ist Mitherausgeber von retten!.

E-Mail: r.kuhnke@drk-ls.de

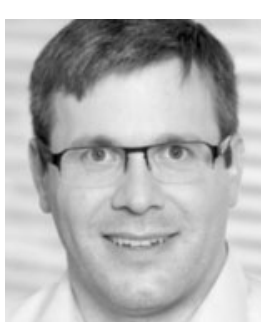

Dr. Wolfgang C. G. von Meißner, MHBA, war bis Ende 2015 Ärztlicher Leiter der DRK-Landesschule Baden-Württemberg und ist ERC-ALS Course Director. Dr. von Meißner ist Facharzt für Anästhesiologie mit den Zusatzbezeichnungen Notfallmedizin und

Intensivmedizin. Zugleich ist er Facharzt für Allgemeinmedizin und arbeitet als Landarzt und Notarzt in Baiersbronn im Schwarzwald. Er ist Mitherausgeber von retten!.

E-Mail: vonmeissner@hausaerzte-am-spritzenhaus.de

Beitrag online zu finden unter http://dx.doi.

org/10.1055/s-0042-112897

Kommentar von Dr. Thomas Jakob, ÄLRD Kreis Herford und Stellv. Vorsitzender der AGNNW

Gerade Notfallsanitäter benötigen Vorgaben eines Handlungskorridors durch Algorithmen und SOPs, um ein Mindestmaß an Rechts- und Handlungssicherheit zu erhalten. In Ostwestfalen-Lippe (OWL) haben wir bereits vor vielen Jahren mit der Erstellung gemeinsamer Algorithmen zu verschiedenen Krankheitsbildern begonnen. Der Vorteil: eine einheitliche Ausbildung und Versorgung einer großen Bevölkerungszahl über die Grenzen von Gebietskörperschaften hinweg, wie sie sonst nur in großen Ballungsräumen möglich ist.

Ausarbeitungen der Ärztlichen Leiter Rettungsdienst (ÄLRD) in OWL fließen in den bundesweiten Pyramidenprozess ein: So werden künftig z. B. SOPs zu invasiven Maßnahmen vom Landesministerium Nordrhein-Westfalen (NRW) und voraussichtlich auch weiteren Bundesländern in die Lehrvorgaben für Notfallsanitäter aufgenommen.

Der abgebildete Algorithmus Schlaganfall zeigt die derzeit gültige Version, die aktuell überarbeitet wird. Grund für die Verzögerung der geplanten Überarbeitung ist die Harmonisierung mit den Rahmen-SOPs, die im Pyramidenprozess kurz vor dem Abschluss stehen. Der noch in diesem Jahr erscheinende überarbeitete Algorithmus wird auch im Layout die Abfolge nach ABCDE-Schema deutlicher hervorheben.

Der Algorithmus verweist auf die grundsätzliche Notarztindikation und Notwendigkeit, bei Störungen definierter Vitalwerte den Notarzt hinzuzuziehen. Er ermöglicht aber die Option des raschen eigenständigen Transports oder im Rendezvous-Verfahren, wenn die abzuarbeitenden Parameter in Ordnung sind und/oder bei inakzeptablen Zeitverlusten durch Wartezeiten. Derzeit wird kontrovers diskutiert, welchen Einfluss die Möglichkeit einer Katheter-Thombektomie auf die Wahl der Zielklinik haben sollte. Hierfür fehlen aber klare Indikatoren.

Autoren der aktuellen Fassung sind die ÄLRD Dr. H. P. Milz (Stadt Bielefeld), B. Strickmann (Kreis Gütersloh), Dr. T. Jakob (Kreis Herford), Dr. M. Gernhardt (Kreis Höxter), I. Christiansen (Kreis Paderborn) und Dr. A. Röper (Studieninstitut Westfalen-Lippe). An der überarbeiteten Version werden sich weitere Gebietskörperschaften (z. B. Kreis Lippe und Stadt Münster) beteiligen. Weitere Algorithmen sind auch im Kompendium der Arbeitsgemeinschaft Notärzte in NRW zu finden, das sich kostenlos über o www.agnnw.de oder im APP-Store herunterladen lässt. 